\title{
Characteristics of magnetised plasma flow around stationary and expanding magnetic clouds
}

\author{
G. Dalakishvili ${ }^{1}$, S. Poedts ${ }^{1}$, H. Fichtner ${ }^{2}$, and E. Romashets ${ }^{3}$ \\ 1 Centre for Plasma Astrophysics, K.U.Leuven, Celestijnenlaan 200B, 3001 Leuven, Belgium \\ e-mail: Giorgi .Dalakishvili@wis.kuleuven.be \\ 2 Institut für Weltraum und Astrophysik, Ruhr-Universität Bochum, Universitätsstrasse 150, 44780 Bochum, Germany \\ 3 Solar Observatory of Prairie View A \& M University, Prairie View, TX 77446, USA
}

Received 6 May 2009 / Accepted 4 September 2009

ABSTRACT

\begin{abstract}
Aims. Studies of interplanetary magnetic clouds have shown that the characteristics of the region ahead of these objects, which are moving away from the Sun in the solar wind, play a role in determining their geo-efficiency, i.e. the kind and the degree of their effects on the Earth environment. Therefore, our main goal is to model and study the plasma parameters in the vicinity of interplanetary magnetic clouds.

Methods. To this end we present a model in which the magnetic clouds are immersed in a magnetised plasma flow with a homogeneous magnetic field. We first calculate the resulting distortion of the external magnetic field and then determine the plasma velocity by employing the frozen-in condition.

Results. Subsequently, the plasma density and pressure are expressed as functions of the magnetic field and the velocity field. Conclusions. The plasma flow parameters are determined by solving the time-independent ideal MHD equations for both the stationary regime and for the case of an expanding cylindrical magnetic cloud, thus extending previous results that appeared in the literature.
\end{abstract}

Key words. magnetohydrodynamics (MHD) - magnetic fields - plasmas - Sun: solar wind

\section{Introduction}

A magnetic cloud (MC) is a specific type of interplanetary coronal mass ejection (see Burlaga 1995; Wimmer-Schweingruber et al. 2006; Démoulin et al. 2008) and can be considered as a magnetically isolated structure moving in the solar wind. There are various studies of MCs, particularly of their inner structure (e.g., Nakwacki et al. 2008), their interaction with the solar wind streams (e.g., Lepping et al. 2008), and their expansion (e.g., Démoulin et al. 2008, and further references therein). While in these investigations the authors mainly deal with the internal cloud dynamics, in the present paper we focus on how an expanding MC perturbs the physical parameters of its ambient environment. In order to enable an analytical description of the structure of both the MC and its immediate surroundings, it has been suggested that MCs are force-free magnetic field configurations (Burlaga 1988; Lepping et al. 1990; Burlaga 1991; Vandas et al. 2006). Numerical studies (De Sterck \& Poedts 1999; De Sterck \& Poedts 2000; Manchester et al. 2004; Chane et al. 2006), exploring the character of magnetised plasma flows near MCs, in which these structures are considered as superconductors (De Sterck \& Poedts 1999; De Sterck \& Poedts 2000), as well as observational data analysis efforts (Owens et al. 2005), have revealed that the region in front of an interplanetary MC has a rather complicated magnetic structure and a complex plasma structure as well. In order to get more insight into the characteristics of this region and the physical phenomena within it, and in order to better understand the geo-efficiency of MCs, an analytical treatment of the magnetised plasma flow in their vicinity is beneficial

In mathematical models of force-free magnetic flux ropes (Burlaga 1988; Lepping et al. 1990; Vandas et al. 2006), the radial component of the magnetic field is zero all throughout the MC including the boundary region. The boundary condition on the MC surface (equal normal components of the magnetic field on both sides) then naturally leads to the requirement that the ambient field must be tangential to the surface of a MC, i.e. the same requirement that is to be satisfied for a superconductor. Analytical expressions for the magnetic field satisfying such boundary conditions can be found by Vandas et al. (2003) and Romashets \& Poedts (2007). In these papers, MCs are considered as superconductors placed in a given ambient magnetic field and the distortion of the external magnetic field has been calculated. First, by Vandas et al. (2003) both spherical and cylindrical shapes of MCs were considered and the existing quantitative models of magnetic field behaviour around MCs were summarised. Second, by Romashets \& Poedts (2007) analytical expressions for the plasma velocity, pressure and density were derived.

In the present study, we consider a MC that is placed in a magnetised plasma flow with an initially homogeneous magnetic field. We assume that the normal component of the magnetic field vanishes at the boundary of the MC and that far from the $\mathrm{MC}$ the magnetic field approaches its undisturbed value. We thus generalise the solutions given by Romashets \& Poedts (2007) for a cylindrical superconductor: both the cases $B_{z}=0$ and $B_{z} \neq 0$ 
are considered (cylindrical coordinates are used). In addition, we present the solution for the magnetic field for the case of a spherical superconductor. We consider solutions for the distortion of the external magnetic field decreasing as $r^{-k}$ (where $r$ is the distance from the axis/centre of the superconductor); where the case $k=3$ leads to the particular solution derived and discussed by Romashets \& Poedts (2007). Finally, we also study the plasma flow parameters around a cylindrical MC that is radially expanding during its evolution in the ambient plasma flow.

The paper is organised as follows: in Sects. 2 and 3 we consider the characteristics of the stationary plasma flow in the case of cylindrical and spherical MCs, respectively. In Sect. 4, the case of an expanding cylindrical MC is investigated. In Sect. 5, we summarise and discuss our results.

\section{Physical parameters of a magnetised plasma flow around a cylindrical superconductor}

In this section, we define two-dimensional distributions of the magnetic, velocity, density and pressure fields in a plasma flow around a cylindrical MC with radius $r_{0}$. Using cylindrical coordinates $(r, \varphi, z)$, we start with constructing a magnetic field that is uniform far from the cylinder (i.e. for $r \gg r_{0}$ ) and tangential to the cylinder surface, i.e. $B_{r}=0$ at $r=r_{0}$, and that has a vanishing $z$-component, i.e. $B_{z}=0$ :

$$
\begin{aligned}
& B_{r}=B_{0} \cos \varphi\left[1-\frac{r_{0}^{k}}{r^{k}}\right], \\
& B_{\varphi}=-B_{0} \sin \varphi\left[1+\frac{(k-1) r_{0}^{k}}{r^{k}}\right], \\
& B_{z}=0 .
\end{aligned}
$$

In order to determine the velocity of the steady state plasma flow around the cylindrical superconductor, the frozen-in condition is used:

$\nabla \times(\boldsymbol{V} \times \boldsymbol{B})=0$.

It is convenient to rewrite Eq. (2.4a) in the following way (see Romashets \& Poedts 2007):

$\boldsymbol{V} \times \boldsymbol{B}=\nabla F$.

From Eq. (2.4b) it then follows that:

$$
\begin{aligned}
-V_{z} B_{\varphi} & =\frac{\partial F}{\partial r}, \\
V_{z} B_{r} & =\frac{1}{r} \frac{\partial F}{\partial \varphi}, \\
V_{r} B_{\varphi}-V_{\varphi} B_{r} & =\frac{\partial F}{\partial z},
\end{aligned}
$$

Equation (2.4b) is solved using a variable separation ansatz, i.e:

$$
F=R(r) \Phi(\varphi) Z(z)
$$

Upon substituting this ansatz in Eqs. (2.5a) and (2.5b) one obtains:

$$
\frac{R^{\prime}}{R} \frac{r\left(r^{k}-r_{0}^{k}\right)}{r^{k}+(k-1) r_{0}^{k}}=\frac{\Phi^{\prime} \tan \varphi}{\Phi}=\text { const. }=C .
$$

Equation (2.7) leads to a set of ordinary differential equations (ODEs) for $R$ and $\Phi$. The solutions of these ODEs are given by:

$R=A_{r} \frac{[x-1]^{C}}{[x]^{\frac{k-1}{k} C}}$.

Where:

$x=\frac{r^{k}}{r_{0}^{k}}$.

After this substitution we get:

$R(r)=A_{r} \frac{\left[r^{k}-r_{0}^{k}\right]^{C}}{r_{0}^{C} r^{(k-1) C}}$

and

$\Phi(\varphi)=A_{\varphi} \sin ^{C} \varphi$

Here, $A_{r}$ and $A_{\varphi}$ are constants. For $Z(z)$ we use the same expression as in paper of Romashets \& Poedts (2007), i.e. $Z=$ $D\left(1+\alpha \sin \frac{z}{z_{0}}\right)$. With $D, \alpha$, and $z_{0}$ constants. This choice satisfies Eqs. (2.5a)-(2.5c).

Equations $(2.5 \mathrm{a})-(2.5 \mathrm{c})$ yield a relation between $F$, the magnetic field and the velocity. Combining Eqs. (2.5c) with Eqs. (2.1), (2.2), (2.8a), and (2.8b) and choosing $C=1$ (see Romashets \& Poedts 2007) we obtain:

$V_{r}=-\frac{r^{k}-r_{0}^{k}}{r^{k}+(k-1) r_{0}^{k}}\left[\alpha V_{0} \frac{r}{z_{0}} \cos \frac{z}{z_{0}}+V_{\varphi} \frac{\cos \varphi}{\sin \varphi}\right]$,

to ensure that $V_{r}$ is finite everywhere, $V_{\varphi}$ should be proportional to $\sin \varphi$. For $V_{\varphi}$, the expression proposed by Romashets \& Poedts (2007) could be used:

$V_{\varphi}=V_{1}^{0}\left(1+\frac{r_{0}}{r}\right) \sin \varphi$

and Eq. (2.5b) gives:

$V_{z}=V_{0}\left[1+\alpha \sin \frac{z}{z_{0}}\right]$.

In general, the magnetic field could be expressed as a linear combination of Eqs. (2.1)-(2.3) i.e.:

$B_{r}=\sum_{k} B_{0 k} \cos \varphi\left[1-\frac{r_{0}^{k}}{r^{k}}\right]$,

$B_{\varphi}=-\sum_{k} B_{0 k} \sin \varphi\left[1+\frac{(k-1) r_{0}^{k}}{r^{k}}\right]$,

$B_{z}=0$.

In the case when $B_{z} \neq 0$, it is possible to express the magnetic field as follows:

$B_{r}=B_{0}\left[1-\frac{r_{0}^{k}}{r^{k}}\right] \frac{\cos \varphi}{1+\frac{z^{2}}{z_{0}^{2}}}$,

$B_{\varphi}=-0.5 B_{0}\left[1+\frac{(k-1) r_{0}^{k}}{r^{k}}\right] \frac{\sin \varphi}{1+\frac{z^{2}}{z_{0}^{2}}}$

$B_{z}=\frac{-0.5 B_{0}}{r}\left[1+\frac{(k-1) r_{0}^{k}}{r^{k}}\right]\left[z_{1}+z_{0} \arctan \frac{z}{z_{0}}\right]$. 
The general form of the magnetic field reads:

$B_{r}=\sum_{k} B_{0 k}\left[1-\frac{r_{0}^{k}}{r^{k}}\right] \frac{\cos \varphi}{1+\frac{z^{2}}{z_{0}^{2}}}$,

$B_{\varphi}=-\sum_{k} 0.5 B_{0 k}\left[1+\frac{(k-1) r_{0}^{k}}{r^{k}}\right] \frac{\sin \varphi}{1+\frac{z^{2}}{z_{0}^{2}}}$,

$B_{z}=\sum_{k} \frac{-0.5 B_{0 k}}{r}\left[1+\frac{(k-1) r_{0}^{k}}{r^{k}}\right]\left[z_{1}+z_{0} \arctan \frac{z}{z_{0}}\right]$.

In analogy with the results obtained by Romashets \& Poedts (2007), we can formulate a relationship between the components of the velocity, namely:

$$
\begin{aligned}
& V_{r} \frac{k+l}{r}\left[\frac{r^{k}+(k-1) r_{0}^{k}}{r^{k}-r_{0}^{k}}\right]+V_{\varphi} 2 l \frac{\cos \varphi}{\sin \varphi} \\
& \quad+V_{z} 2 k \frac{1}{\left(z_{1}+z_{0} \arctan \frac{z}{z_{0}}\right)\left(1+\frac{z^{2}}{z_{0}^{2}}\right)}=0 .
\end{aligned}
$$

In order to determine the plasma pressure $P$ and the density $\varrho$ of the plasma flow, in addition to Eqs. (2.1)-(2.4) we should consider the equation of motion, the continuity equation, and the equation of state. These equation read, respectively:

$\varrho(\boldsymbol{V} \cdot \nabla) \boldsymbol{V}+\nabla\left(P+\frac{B^{2}}{8 \pi}\right)=\frac{(\boldsymbol{B} \cdot \nabla) \boldsymbol{B}}{4 \pi}$,

$\nabla \cdot(\varrho \boldsymbol{V})=0$,

and

$\boldsymbol{V} \cdot \nabla\left(\frac{P}{\varrho^{\gamma}}\right)=0$.

Here $\gamma$ corresponds to the specific heat ratio. From the system of Eqs. (2.1)-(2.4) and (2.18)-(2.20), the expressions for the plasma density and pressure can be derived (see Romashets \& Poedts 2007):

$\varrho=-\frac{1}{8 \pi} \frac{\boldsymbol{V} \cdot \nabla \frac{\boldsymbol{V} \cdot \boldsymbol{B} \times(\nabla \times \boldsymbol{B})}{\nabla \cdot \boldsymbol{V}}+\gamma \boldsymbol{V} \cdot \boldsymbol{B} \times(\nabla \times \boldsymbol{B})}{\boldsymbol{V} \cdot \nabla \frac{\boldsymbol{V} \cdot \boldsymbol{V} \cdot \nabla) \boldsymbol{V}}{\nabla \cdot \boldsymbol{V}}+(\gamma-1) \boldsymbol{V} \cdot(\boldsymbol{V} \cdot \nabla) \boldsymbol{V}}$,

and

$$
\begin{aligned}
P= & -\frac{1}{8 \pi} \frac{\boldsymbol{V} \cdot(\boldsymbol{V} \cdot \nabla) \boldsymbol{V}}{\gamma \nabla \cdot \boldsymbol{V}} \frac{\boldsymbol{V} \cdot \nabla \frac{\boldsymbol{V} \cdot \boldsymbol{B} \times(\nabla \times \boldsymbol{B})}{\nabla \cdot \boldsymbol{V}}+\gamma \boldsymbol{V} \cdot \boldsymbol{B} \times(\nabla \times \boldsymbol{B})}{\boldsymbol{V} \cdot \nabla \frac{\boldsymbol{V} \cdot(\boldsymbol{V} \cdot \nabla) \boldsymbol{V}}{\nabla \cdot \boldsymbol{V}}+(\gamma-1) \boldsymbol{V} \cdot(\boldsymbol{V} \cdot \nabla) \boldsymbol{V}} \\
& +\frac{\boldsymbol{V} \cdot \boldsymbol{B} \times(\nabla \times \boldsymbol{B})}{8 \pi \nabla \cdot \boldsymbol{V}} .
\end{aligned}
$$

Note, however, that the obtained mathematical solutions for the plasma pressure $P$ and the plasma density $\varrho$, can become negative and thus loose their physical meaning. In order to obtain physically correct solutions for each value of $k$, proper values of the free parameters should be chosen. For the parameter value $k=2$, these plasma quantities become zero.

Figures 1 and 2 illustrate how the magnitude of the parameter $k$ influences the character of the plasma flow and the magnetic field around the MC. In Fig. 1, in panels a) to d) the streamlines and the magnetic field lines are displayed for the different cases $k=3,5,7$, and 20 , respectively. This figure illustrates that as the value of $k$ is increased, the shape of both the streamlines and
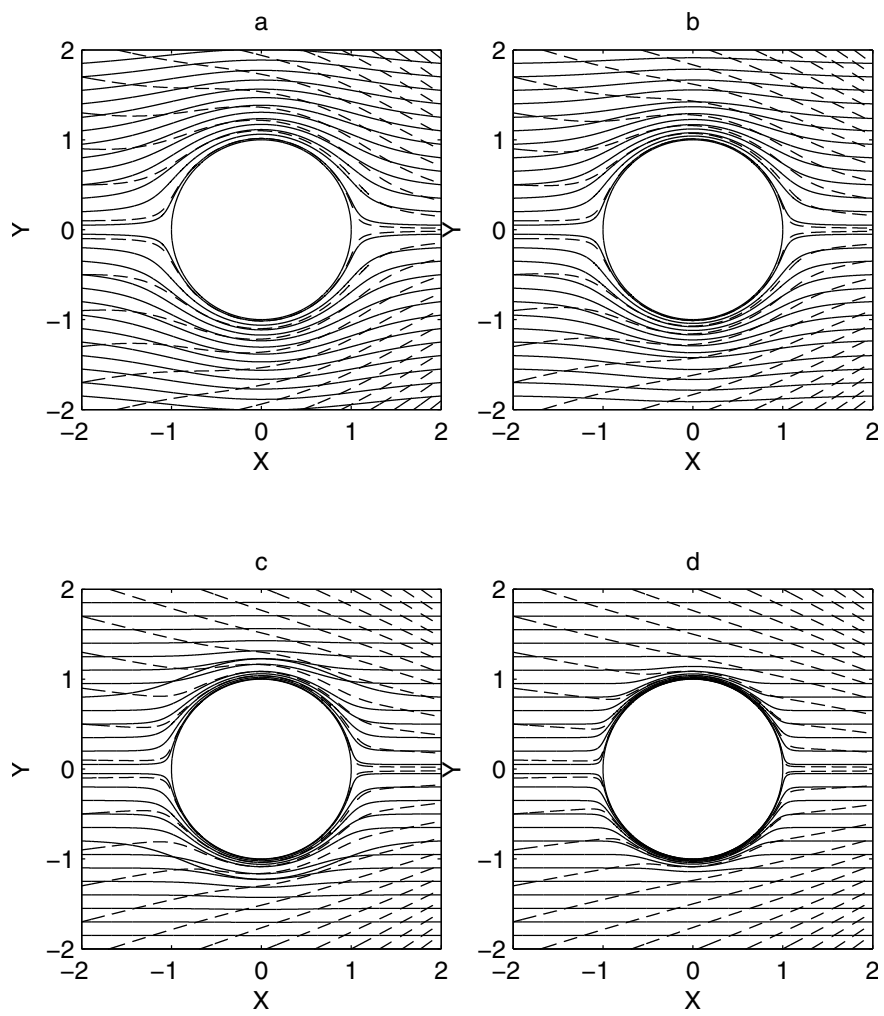

Fig. 1. Distribution of the plasma velocity stream lines and magnetic field lines around cylindrical MC, in the plane $z=0$ and with $B_{z}=0$. The dashed lines correspond to streamlines and the solid lines represent magnetic field lines. Panel a) corresponds to the case when $k=3$; panel b) corresponds to the case $k=5$; panel c) shows the case $k=7$; and panel d) corresponds to $k=20$.
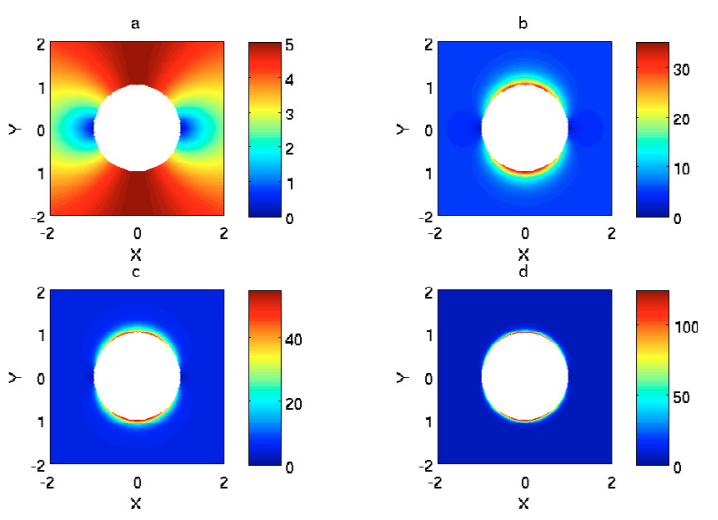

Fig. 2. Contour plots of the magnitude of the magnetic field around cylindrical MC in the plane $z=0$ for $B_{0}=5 \mathrm{nT}, B_{z}=0$. Panel a) corresponds to the case $k=3$; panel b) represents the case $k=7$; panel c) shows the case $k=11$ and panel d) corresponds to the case $k=25$. The magnetic field $B$ is given in nT.

the magnetic field lines changes and both gradually evolve into straight lines, except of course in the immediate neighborhood of the MC where the flow and the magnetic field are both deflected around the MC (due to the imposed boundary conditions).

In Fig. 2 we plot the strength of magnitude of the magnetic field for $B_{0}=5 \mathrm{nT}$ and for the cases for $k=3,7,11$, and 25, in the panels a) to d), respectively. This figure clearly demonstrates that for increasing values of the model parameter $k$, the magnetic field becomes ever more uniform in the surroundings of the cylinder and its value increases in the immediate vicinity of 


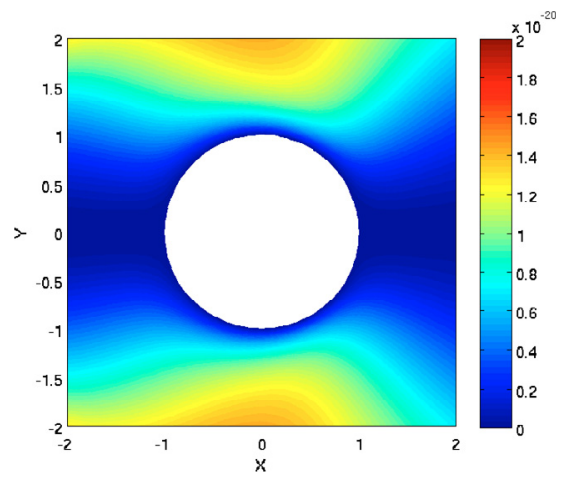

Fig. 3. Contour plots of the magnitude of the plasma density around cylindrical MC in the plane $z=0$ for the parameter values $V_{0}=$ $22.5 \mathrm{~km} \mathrm{~s}^{-1}, V_{1}^{0}=-7.5 \mathrm{~km} \mathrm{~s}^{-1}, z=0, z_{0}=r_{0}$, and $\alpha=0.3, k=1$. Density is given in $\mathrm{kg} / \mathrm{m}^{3}$.

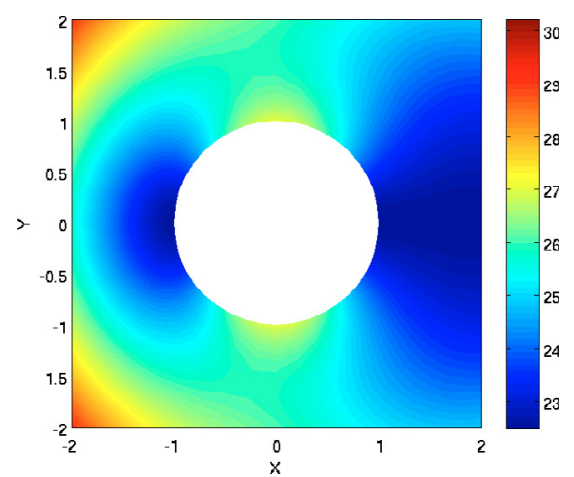

Fig. 4. Contour plots of the magnitude of the plasma velocity around cylindrical $\mathrm{MC}$ in the plane $z=0$ for the parameter values $V_{0}=$ $22.5 \mathrm{~km} \mathrm{~s}^{-1}, V_{1}^{0}=-7.5 \mathrm{~km} \mathrm{~s}^{-1}, z=0, z_{0}=r_{0}$, and $\alpha=0.3, k=1$. Velocity is given in $\mathrm{km} \mathrm{s}^{-1}$.

the MC surface (in Fig. 2 one can see dark red region indicating a comparably strong magnetic field).

Figures 3 and 4 present contour plots of the plasma density $\varrho$ and the plasma velocity $V$ for $k=1, V_{0}=22.5 \mathrm{~km} \mathrm{~s}^{-1}, V_{1}^{0}=$ $-7.5 \mathrm{~km} \mathrm{~s}^{-1}, z=0, z_{0}=r_{0}$, and $\alpha=0.3$. One can see that the density decreases just in front and behind of the MC.

\section{Magnetic field disturbance around a spherical magnetic cloud}

In the present section, we define a three dimensional magnetic field in a plasma flow around a spherical superconductor with radius $r_{0}$. We consider a three-dimensional magnetic field satisfying the same boundary conditions as introduced in the previous section:

$$
\begin{aligned}
& B_{r}=-B_{0} \cos \varphi \sin \theta\left[1-\frac{r_{0}^{k}}{r^{k}}\right], \\
& B_{\theta}=-B_{0} \cos \varphi \cos \theta\left[1+\left(\frac{k-2}{2}\right) \frac{r_{0}^{k}}{r^{k}}\right], \\
& B_{\varphi}=B_{0} \sin \varphi\left[1+\left(\frac{k-2}{2}\right) \frac{r_{0}^{k}}{r^{k}}\right] .
\end{aligned}
$$

Note that we now used spherical coordinates denoted as usual by $(r, \theta, \varphi)$. In order to determine the steady state velocity field of the flow around the spherical superconductor, the frozen-in condition is used again:

$\nabla \times(\boldsymbol{V} \times \boldsymbol{B})=0$.

It is convenient to rewrite Eq. (3.2a) as follows (Romashets \& Poedts 2007):

$\boldsymbol{V} \times \boldsymbol{B}=\nabla F$.

From Eq. (3.2a) it follows that

$\boldsymbol{B} \cdot \nabla F=0$,

which shows that $F$ is constant along the magnetic field lines.

Equation (3.2b) leads to a relation between $F$, the magnetic field and the velocity:

$$
\begin{aligned}
V_{\theta} B_{\varphi}-V_{\varphi} B_{\theta} & =\frac{\partial F}{\partial r}, \\
V_{\varphi} B_{r}-V_{r} B_{\varphi} & =\frac{1}{r} \frac{\partial F}{\partial \theta} \\
V_{r} B_{\theta}-V_{\theta} B_{r} & =0 .
\end{aligned}
$$

After the definition of the magnetic field $\boldsymbol{B}$ and choosing a proper value for $F$, solving the system of Eqs. (3.3a)-(3.3c) will give us an expression for the velocity field $\boldsymbol{V}$.

In general, the magnetic field is a linear combination of Eqs. (3.1a)-(3.1c):

$$
\begin{aligned}
& B_{r}=-\sum_{k} B_{0 k} \cos \varphi \sin \theta\left[1-\frac{r_{0}^{k}}{r^{k}}\right], \\
& B_{\theta}=-\sum_{k} B_{0 k} \cos \varphi \cos \theta\left[1+\left(\frac{k-2}{2}\right) \frac{r_{0}^{k}}{r^{k}}\right], \\
& B_{\varphi}=\sum_{k} B_{0 k} \sin \varphi\left[1+\left(\frac{k-2}{2}\right) \frac{r_{0}^{k}}{r^{k}}\right] .
\end{aligned}
$$

\section{Magnetic field and plasma velocity distribution around expanding cylindrical magnetic clouds}

In this section, we define the magnetic field and the plasma flow velocity around an expanding MC. We consider a cylindrical MC with radius $r_{0}$. In previous studies, (Shimazu \& Vandas 2002) and (Farrugia et al. 1995) considered the dynamics of the physical parameters inside the $\mathrm{MC}$ as a flux rope. In the present paper, on the contrary, we consider the perturbation of the plasma parameters outside the MC. It is assumed that the cylindrical MC expands radially, in a cylindrical symmetric manner i.e., $r_{0}=r_{0}(t)$, where $r_{0}(t)$ is a certain function of time.

Let us first determine the corresponding magnetic field. The magnetic field should be tangential to the MC surface (i.e. $B_{r}=0$ at $r_{0}$ ) and uniform far from the cylinder, so for $r \gg r_{0}$ ). The components of the magnetic field that satisfies these conditions can be expressed in the following way:

$$
\begin{aligned}
& B_{r}=B_{0} \cos \varphi\left[1-\frac{r_{0}^{k}}{r^{k}}\right], \\
& B_{\varphi}=-B_{0} \sin \varphi\left[1+\frac{(k-1) r_{0}^{k}}{r^{k}}\right],
\end{aligned}
$$

$B_{z}=0$. 
Here, we now have

$r_{0}=r_{0}(t)$

and

$B_{0}=B_{0}(t)$

From Faraday's law we obtain

$\nabla \times[\boldsymbol{V} \times \boldsymbol{B}]=\frac{\partial \boldsymbol{B}}{\partial t}$

This then leads to the following equations:

$$
\begin{aligned}
\nabla \times[\boldsymbol{V} \times \boldsymbol{B}]_{r}= & -B_{0} \cos \varphi k r^{-k} r_{0}^{k-1} \dot{r}_{0}+\dot{B}_{0} \cos \varphi\left[1-\frac{r_{0}^{k}}{r^{k}}\right], \\
\nabla \times[\boldsymbol{V} \times \boldsymbol{B}]_{\varphi}= & -B_{0} \sin \varphi k(k-1) r^{-k} r_{0}^{k-1} \dot{r}_{0} \\
& -\dot{B}_{0} \sin \varphi\left[1+\frac{(k-1) r_{0}^{k}}{r^{k}}\right],
\end{aligned}
$$

and

$\nabla \times[\boldsymbol{V} \times \boldsymbol{B}]_{z}=0$.

For further considerations, it is convenient to introduce a new vector field $\boldsymbol{A}$ :

$\boldsymbol{V} \times \boldsymbol{B} \equiv \boldsymbol{A}$.

We assume symmetry along the cylindrical axis, this means:

$\frac{\partial}{\partial z}=0$

A combination of Eqs. (4.6)-(4.10) then yields the related equations for the components of the vector potential $\boldsymbol{A}$ :

$[\nabla \times \boldsymbol{A}]_{r}=\frac{1}{r} \frac{\partial A_{z}}{\partial \varphi}$

$[\nabla \times \boldsymbol{A}]_{\varphi}=-\frac{\partial A_{z}}{\partial r}$

and

$[\nabla \times \boldsymbol{A}]_{z}=\frac{1}{r} \frac{\partial r A_{\varphi}}{\partial r}-\frac{1}{r} \frac{\partial A_{r}}{\partial \varphi}$.

The component $A_{z}$, which satisfies Eqs. (4.11)-(4.13), can be expressed as:

$A_{z}=-B_{0} k r^{1-k} \sin \varphi r_{0}^{k-1} \dot{r}_{0}+\dot{B}_{0} r \sin \varphi\left[1-\frac{r_{0}^{k}}{r^{k}}\right]$.

From Eq. (4.9), the equations for the components of the velocity can then be determined:

$\nabla \times[\boldsymbol{V} \times \boldsymbol{B}]_{r}=A_{r}=-V_{z} B_{\varphi}$,
$\nabla \times[\boldsymbol{V} \times \boldsymbol{B}]_{\varphi}=A_{\varphi}=-V_{z} B_{r}$,

and

$\nabla \times[\boldsymbol{V} \times \boldsymbol{B}]_{z}=A_{z}=V_{r} B_{\varphi}-V_{\varphi} B_{r}$
Equations (4.1)-(4.3) and (4.17) then finally lead to an expression for the radial velocity component $V_{r}$ :

$$
\begin{aligned}
V_{r}= & -\frac{1-\frac{r_{0}^{k}}{r^{k}}}{1+(k-1) \frac{r_{0}^{k}}{r^{k}}} V_{\varphi} \frac{\cos \varphi}{\sin \varphi}+k \frac{\frac{r_{0}^{k}}{r^{k}} \dot{r}_{0}}{1+(k-1) \frac{r_{0}^{r^{k}}}{r^{k}}} \\
& +\frac{\dot{B}_{0}}{B_{0}} r\left[\frac{r^{k}-r_{0}^{k}}{r^{k}+(k-1) r_{0}^{k}}\right] .
\end{aligned}
$$

From Eq. (4.18) we can see that at $r=r_{0}$ the radial component of velocity $V_{r}=\dot{r}_{0}$. This simply means that, at the surface of the $\mathrm{MC}$, the radial velocity is equal to the expansion velocity of the MC's surface. In other words, the external plasma flow velocity is tangential to the MC's surface. In order to provide finite values of $V_{r}$ at $\varphi=0, V_{\varphi}$ should be a function that is proportional to $\sin \varphi$. For $V_{\varphi}$ one can choose the same value as given in the paper Romashets \& Poedts (2007). The simplest solution for $A_{r}$ and $A_{\varphi}$, satisfying Eqs. (4.8), (4.9), and (4.13) is:

$A_{r}=A_{\varphi}=0$.

Equations (4.15), (4.16), and (4.19) then yield that $V_{z}=0$. This solution could be generalised: any function $A+\nabla F$ will satisfy Eq. (5). The potential $F$ could be the same function as the choice discussed by Romashets \& Poedts (2007).

In order to ensure conservation of magnetic flux, the following functions are chosen:

$r_{0}(t)=\left(R_{0}\left(R_{0}+V_{E}\left(t-t_{0}\right)\right)\right)^{1 / 2}$,

and

$B_{0}(t)=R_{0} b_{0} /\left(R_{0}+V_{E}\left(t-t_{0}\right)\right)$.

Here $R_{0}$ corresponds to the radius of the $\mathrm{MC}$ at time $t=t_{0}$, and $b_{0}$ denotes the unperturbed magnetic field at the same moment in time. Note that $V_{E}$ has the same order as the MC's expansion velocity and the values of this velocity are presented in Lepping et al. (2008).

\section{Conclusions}

In this paper, we first generalised the steady (time-independent) solutions for the magnetic field and plasma flow velocity around a cylindrical MC as derived by Romashets \& Poedts (2007) by considering more general distortions of the ambient magnetic field. While in the study by Romashets \& Poedts (2007) the authors considered the case of a distortion of the ambient magnetic field decreasing with $r^{-3}$ from the MC axis, in the present paper we investigated a class of magnetic field distortions decreasing with $r^{-k}$, where the parameter $k$ determines the strength of the influence of the considered MC on the surrounding ambient magnetic field. We illustrated the distribution of the plasma density around the $\mathrm{MC}$ using the formulas given by Romashets \& Poedts (2007). We remarked, however, that care has to be taken because not every set of free parameters leads to a physically consistent solution, and we found the right values for $V_{0}$ and $V_{1}^{0}$ in the case of $k=1$.

The higher the value of $k$, the smaller or the less extensive the distortion around the MC. We calculated and illustrated how the value of $k$ influences the configuration and magnitude of the magnetic field as well as the velocity field around a cylindrical MC treated as a superconductor (see Figs. 1, 2).

Note that different values of the model parameter $k$ correspond to different current regimes. For example, when $k=2$ the plasma flow around a cylindrical superconductor with $B_{z}=0$ 
is current free. The same is true around a spherical superconductor, another extension of the previous analysis we studied as well. A third extension we considered involved a study of the character of the plasma flow parameters around a radially expanding cylindrical MC, instead of a steady flow around an MC with fixed radius.

These results can, on the one hand, be used as a starting point for an investigation of the interactions of interplanetary MCs with the ambient plasma flows and the characterisation of the forces acting on MCs or their surfaces. On the other hand, the new analytical expressions will serve as initial conditions for more involved numerical simulations, which will enable a study of the much more complicated dynamics of plasma flows around non-cylindrical and/or non-spherical MCs. Such numerical simulations are expected to enable us to investigate the stability of the time-independent solutions. Moreover, while we here presented solutions of the MHD equations for the region outside the MCs by prescribing general magnetic field configurations, the mentioned numerical studies will eventually allow us to construct self-consistent solutions also for the region inside the MC.

Acknowledgements. These results were obtained in the framework of the projects GOA/2009-009 (K.U.Leuven), G.0304.07 (FWO-Vlaanderen) and C 90347 (ESA Prodex 9). Financial support by the European Commission through the SOLAIRE Network (MTRN-CT-2006-035484) and by the Georgian National Science Foundation grant GNSF/ST06/4-096 is gratefully acknowledged. The work also benefitted from the Forschergruppe 1048 (project FI 706/81) supported by the Deutsche Forschungsgemeinschaft (DFG).

\section{References}

Burlaga, L. F. 1988, J. Geophys. Res., 93, 7217

Burlaga, L. F. 1991, Physics of the inner heliosphere 2, Particles, waves and turbulence, XI (Berlin Heidelber New York: Springer-Verlag), 352

Burlaga, L. F. 1995, Interplanetary Magnetohydrodynamics (New York: Oxford University Press)

Chane, E., Van der Holst, B., Jacobs, C., Poedts, S., \& Kimpe, D. 2006, A\&A, 447, 727

Démoulin, P., Nakwacki, M. S., Dasso, S., \& Mandrini, S. H. 2008, Sol. Phys., 250,347

Farrugia, C. J., Osherovich, V. A., \& Burlaga, L. F. 1995, Ann. Geophys., 13, 815

Lepping, R. P., Burlaga, L. F., \& Jones, J. A. 1990, J. Geophys. Res., 95, 11957

Lepping, R. P., Wu, C. C., Berdichevsky, D. B., \& Ferguson, T. 2008, Ann. Geophys., 26, 1919

Manchester, W. B., Gombosi, T. I., Roussev, I., et al. 2004, J. Geophys. Res., 109, A01102

Nakwacki, M., Dasso, S., Mandrini, C., \& Démoulin, P. 2008, J. Atmos. Sol. Terr. Phys., 70, 1318

Owens, J. M., Cargill, P. J., Pagel, C., Siscoe, G. L., \& Crooker, N. U. 2005, J. Geophys. Res., 110

Romashets, E., \& Poedts, S. 2007, A\&A, 475, 1093

De Sterck, H., \& Poedts, S. 1999, A\&A, 343, 641

De Sterck, H., \& Poedts, S. 2000, Phys. Rev. Lett., 84, 5524

Shimazu, H., \& Vandas, M. 2002, EP\&S, 54, 783

Vandas, M., Romashets, E. P., \& Watari, S. 2003, A\&A, 412, 281

Vandas, M., Romashets, E. P., Watari, S., et al. 2006, Adv. Spa. Res., 38, 441

Wimmer-Schweingruber, R. F., Crooker, N. U., Balogh, A., et al. 2006, Space Sci. Rev., 123, 177 